\title{
A clinical study on the value of carotid cistern drainage during intracranial aneurysm clipping
}

\author{
Guo-Liang Jin, Zi-Gang Yuan, Yong-Zhi Zhou, Jian-Li Wang
}

Department of Neurosurgery, Shaoxing People's Hospital (Shaoxing Hospital, Zhejiang University School of Medicine), Shaoxing, Zhejiang Province, China

Submitted: 27 March 2020

Accepted: 16 May 2020

Arch Med Sci

DOI: https://doi.org/10.5114/aoms.2020.98960

Copyright $\odot 2020$ Termedia \& Banach

\section{Abstract}

Introduction: To investigate the value of carotid cistern drainage in patients with aneurysmal subarachnoid haemorrhage during intracranial aneurysm clipping.

Material and methods: Patients with ruptured intracranial aneurysms were included in this study. During the operation, the craniotomy was performed via a pterional approach to separate the Sylvian cistern and carotid cistern. After completing the clipping of the intracranial aneurysm, a drainage tube was placed in the carotid cistern between the ipsilateral internal carotid artery and optic nerve or lateral internal carotid artery, which was led out through the dura mater, cranial flap foramen or gap and scalp, and connected with an external drainage device.

Results: A total of 6 patients with aneurysmal subarachnoid haemorrhage, who required craniotomy for clipping, were enrolled in the study. These patients were given carotid cistern drainage during the operation. Among these 6 patients, 2 had anterior communicating aneurysms, 1 had a posterior communicating aneurysm of the internal carotid artery, 2 had middle cerebral artery furcation aneurysms, and 1 patient had multiple intracranial aneurysms. For the Hunt-Hess score before the operation, 5 patients were in grade II and 1 patient was in grade III. Furthermore, the postoperative drainage was unobstructed, with a daily drainage of 130-290 ml of haemorrhagic cerebrospinal fluid (CSF).

Conclusions: The CSF drainage of the carotid cistern applied in patients with aneurysmal subarachnoid haemorrhage during the craniotomy for clipping can effectively remove the subarachnoid haemorrhage, control the intracranial hypertension, and reduce the incidence of cerebral vasospasm and hydrocephalus.

Key words: intracranial aneurysm, aneurysmal subarachnoid haemorrhage, neurosurgical clipping, carotid cistern, GOS scores.

\section{Introduction}

The treatment of subarachnoid haemorrhage caused by a ruptured intracranial aneurysm can be divided into two parts: (1) prevention of the recurrence of aneurysm rupture; (2) prevention and treatment of a series of reactions caused by increased intracranial pressure, cerebral vasospasm, and hydrocephalus after the subarachnoid haemorrhage [1, 2]. There are two major approaches to prevent the recurrence of aneurysms from rupture: endovascular coil occlusion and craniotomy for aneurysm clipping. Although endovascular interventional treatment has developed

\author{
Corresponding author: \\ Jian-Li Wang \\ Department \\ of Neurosurgery \\ Shaoxing People's Hospital \\ Shaoxing Hospital \\ Zhejiang University \\ School of Medicine \\ 568 Zhongxing \\ North Road \\ Shaoxing 312000 \\ Zhejiang Province \\ China \\ Phone: +86 57588228888 \\ Fax: +86 57585138402 \\ E-mail: wangj|neur@163.com
}


rapidly and it is definitely effective, some intracranial aneurysms are not suitable for interventional therapy or cannot achieve satisfactory results through interventional therapy. The traditional method of craniotomy for aneurysm clipping is still widely used [3, 4]. In the aspect of clearing the subarachnoid haemorrhage, lumbar cistern drainage is a common and effective method [5], either after endovascular coil occlusion or after craniotomy for aneurysm clipping. However, for patients with intracranial hypertension or intracranial haematoma, lumbar cistern drainage may carry a risk of inducing transforamen magna herniation.

Furthermore, the value of carotid cistern drainage during intracranial aneurysm clipping remains unknown. Therefore, we have conducted this study to investigate the clinical significance of carotid cistern drainage in patients with aneurysmal subarachnoid haemorrhage during intracranial aneurysm clipping.

\section{Material and methods}

\section{Study subjects}

During the craniotomy and after the aneurysm was clamped, a drainage tube was placed in the carotid cistern to drain the haemorrhagic cerebrospinal fluid (CSF). It is easy to operate and effective, and it deserves clinical attention. Therefore, the cases of CSF drainage of carotid cistern during intracranial aneurysm clipping in our hospital from January 2019 to March 2019 were analysed in the present study. Furthermore, the present study aims to preliminarily explore the clinical significance of carotid cistern drainage in intracranial aneurysm clipping in patients with aneurysmal subarachnoid haemorrhage.

Therefore, patients with ruptured intracranial aneurysms were enrolled in the present study as the main research subjects. Placement of a carotid cistern drainage tube was planned to facilitate the drainage and reduce the intracranial pressure after completing intracranial aneurysm clipping during the operation. The present study was carried out in accordance with the Helsinki Declaration of the World Medical Association and approved by the Ethics Committee of the hospital. All patients provided a signed informed consent form.

\section{Inclusion and exclusion criteria}

Inclusion criteria: (1) patients with a confirmed diagnosis of ruptured intracranial aneurysm, who required intracranial aneurysm clipping; (2) patients who provided a signed informed consent form. Exclusion criteria: (1) patients who suffered from advanced malignant tumours; (2) patients or their family members who refused the surgical treatment; (3) patients who suffered from severe coagulation dysfunction; (4) patients who did not have complete clinical data.

\section{Operation methods}

According to the location of the aneurysm, on the left or right, a pterional approach was chosen for the craniotomy. A dominant blood supply approach was picked up for the anterior communicating aneurysm according to the digital subtraction angiography (DSA). The Sylvian cistern and carotid cistern were separated to release the CSF. The aneurysm neck was separated, and the aneurysm was clamped. The parent artery and its main branches were confirmed to be unobstructed. A drainage tube (lumbar cistern drainage tube, model: 27302, Medtronic) was inserted, approximately $10 \mathrm{~mm}$ in depth. It was led out through the dura mater, cranial flap foramen/gap and scalp, then connected with an external drainage device. An intracranial pressure monitoring probe was placed under the dura mater, and an extracranial subcutaneous drainage tube was placed. The pressure of the carotid cistern drainage was managed at 80-180 $\mathrm{mm} \mathrm{Hg}$. The drainage tube was removed after 24-72 h. The extracranial subcutaneous drainage tube was removed 24-48 h after the operation.

An injection of $20 \%$ mannitol and another of $10 \%$ glycerol fructose were used to reduce the intracranial pressure before surgery. Nimodipine injection was given for the prevention and treatment of cerebral vasospasm before surgery. Antibiotics were used to prevent infection during the craniotomy. After the surgery, the patients received the fluid replacement, $20 \%$ mannitol injection, $10 \%$ glycerol fructose for the reduction of the intracranial pressure according to the monitoring results of intracranial pressure. After the surgery, nimodipine injection was continued for 1 to 2 weeks. After 2 weeks, nimodipine tablets were taken orally until the symptoms of cerebral vasospasm were relieved.

\section{Observation index}

The patient's consciousness status, Glasgow Coma Scale (GCS) scores, intracranial pressure monitoring (ICP), Numeric Rating Scale (NRS) of pain, and daily drainage volume of the carotid cistern were observed after operation. Then, the routine head computed tomography (CT) was reviewed on the second day after operation, the DSA was reviewed between 2 weeks and 2 months, and clinical follow-ups were carried out for 3-6 months.

\section{Statistical analysis}

SPSS 20.0 statistical software was used to process the data in the present study. Measurement 
data were expressed as mean \pm standard deviation (mean \pm SD) while enumeration data were expressed as percentages (\%). $P<0.05$ was considered statistically significant.

\section{Results}

\section{General data}

From January 2019 to March 2019, a total of 6 patients with aneurysm rupture and subarachnoid haemorrhage, who required craniotomy for aneurysm clipping with carotid cistern drainage, were enrolled in the present study, including two males and four females, and their age ranged within 49-69 years, with a mean age of 59.8 years. For the Hunt-Hess scale on the day of the operation, 5 patients were in grade II and one patient was in grade III. Sites of the aneurysms: 2 patients had anterior communicating aneurysms, 1 patient had a posterior communicating aneurysm of the internal carotid artery, 2 patients had middle cerebral artery furcation aneurysms, and 1 patient had multiple aneurysms (one posterior communicating aneurysm each in the bilateral internal carotid arteries, the left anterior cho- roidal artery, and the furcation of bilateral middle cerebral arteries; and two anterior communicating aneurysms). The preoperative imaging data of these patients are presented in Figure 1.

\section{Clinical efficacy of carotid cistern drainage after operation}

The drainage tubes of the carotid cistern were all unobstructed in 6 patients after operation, with an average daily drainage volume of $130-290 \mathrm{ml}$. No blockage of the drainage tube occurred within 24-72 h, no CSF leakage was found at the incision after the extubation, and no intracranial infection was reported. During the carotid cistern drainage, the median intracranial pressure of patients was 6-16 $\mathrm{mm} \mathrm{Hg}$, and the pain number scores were within 1-6 points (Table I). The patient's intraoperative condition is presented in Figure 2.

\section{Follow-up and prognosis of patients after} carotid cistern drainage

The postoperative follow-up lasted for 3-6 months. Six patients did not have hydrocephalus and recovered well. The Glasgow Outcome Scale

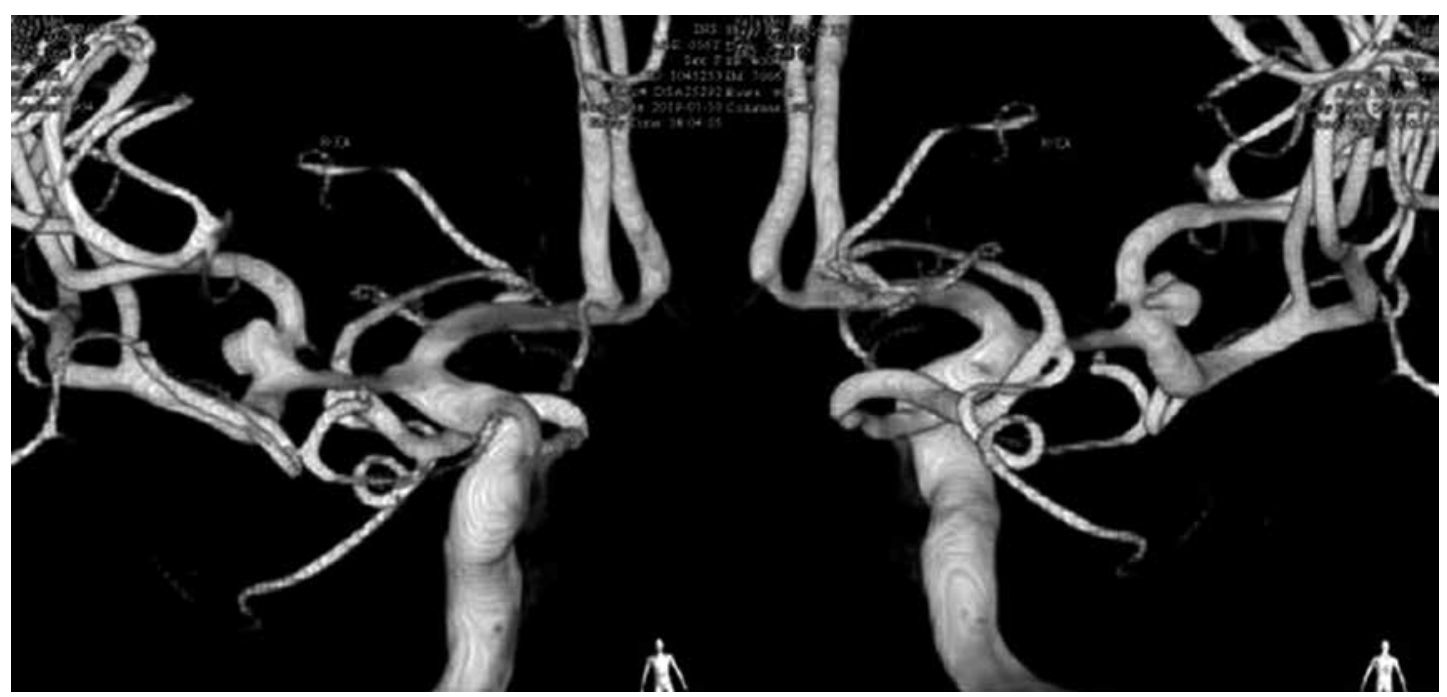

Figure 1. An aneurysm at the furcation of the right middle cerebral artery in preoperative DSA (3D reconstruction)

Table I. Clinical efficacy of carotid cistern drainage after the operation

\begin{tabular}{|lccccc|}
\hline No. & Gender & Age [years] & Aneurysm site & $\begin{array}{c}\text { Mean daily drainage volume } \\
{[\mathrm{ml}]}\end{array}$ & NRS \\
\hline 1 & Female & 57 & $\begin{array}{c}\text { Right middle cerebral artery } \\
\text { occlusion }\end{array}$ & 290 & 3 \\
\hline 2 & Male & 49 & Anterior communicating & 130 & 6 \\
\hline 3 & Female & 67 & Multiple aneurysms & 70 & 2 \\
\hline 4 & Female & 59 & Anterior communicating & 130 & 3 \\
\hline 5 & Female & 58 & Right posterior communicating & 280 & 2 \\
\hline 6 & Male & 69 & $\begin{array}{c}\text { Left middle cerebral artery } \\
\text { furcation }\end{array}$ & 180 & 3 \\
\hline
\end{tabular}



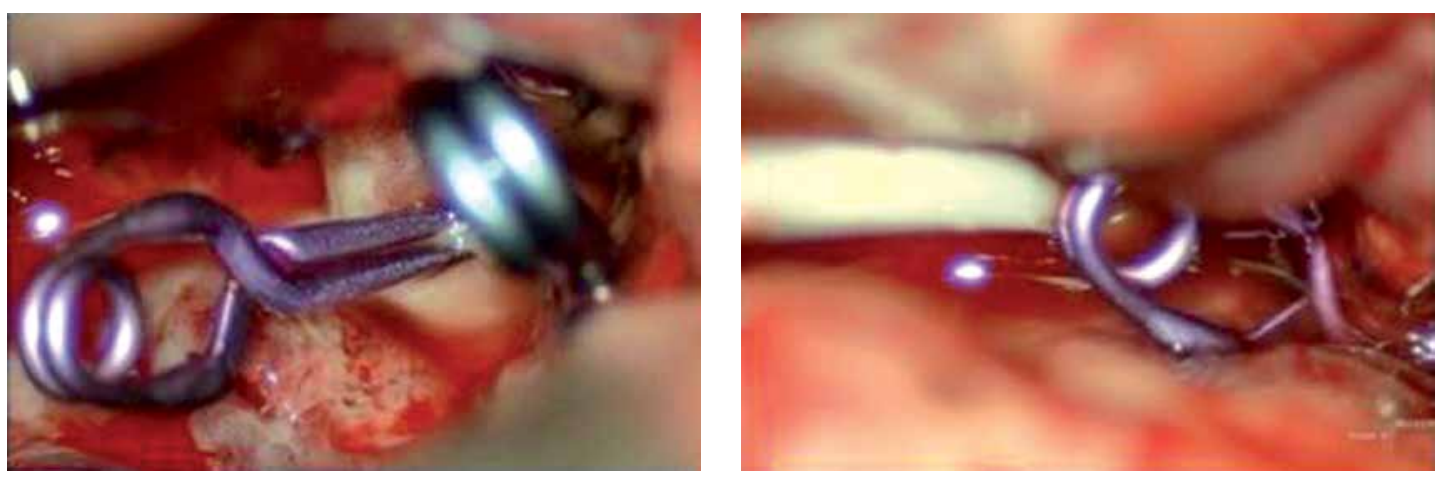

Figure 2. The postoperative aneurysm clipping and carotid cistern drainage tube in intraoperative microscopic findings
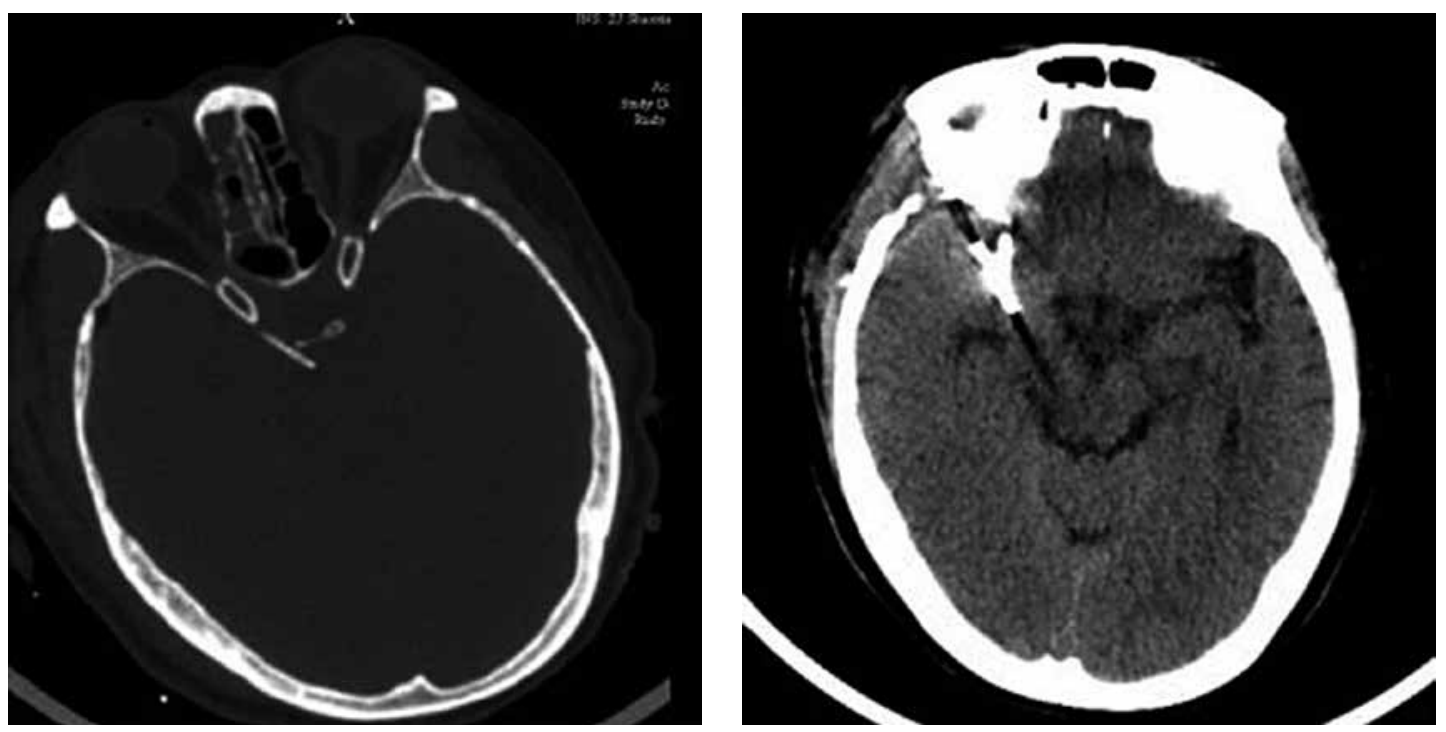

Figure 3. The right carotid cistern drainage tube and aneurysm clipping in postoperative CT review

(GOS) scores were in grade $\mathrm{V}$, means grade 5 . The imaging data during the postoperative follow-up are presented in Figures 3 and 4.

\section{Discussion}

The study results reveal that the drainage tubes were unobstructed after carotid cistern

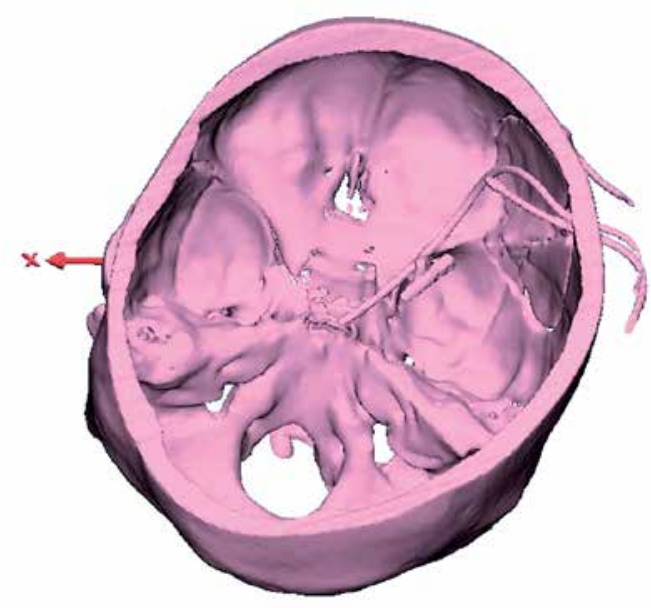

Figure 4. Postoperative review of DSA drainage in 6 patients with aneurysmal subarachnoid haemorrhage requiring craniotomy for aneurysm clipping. The patients have produced 130-290 $\mathrm{ml}$ of haemorrhagic CSF daily, with no blockage of the drainage tube, no CSF leakage at the incision, and no occurrence of intracranial infection and hydrocephalus. The median intracranial pressure was within 6-16 $\mathrm{mm} \mathrm{Hg}$ during the drainage. The postoperative follow-up lasted for 3-6 months, and all patients recovered well, with a GOS score of 5 .

The rupture of intracranial aneurysms can lead to subarachnoid haemorrhage or intracerebral haematoma formation. It is a common issue to control the intracranial hypertension and prevent the cerebral vasospasm and hydrocephalus after the intravascular interventional treatment of aneurysms or craniotomy for aneurysm clipping [6]. However, this is sometimes difficult to predict, and the treatment outcome is not good [7]. After dealing with aneurysm rebleeding, it is very important to remove the subarachnoid haemorrhage in time, which can effectively reduce the stimulation of haemorrhagic CSF, prevent the cerebral 
vasospasm, control the intracranial hypertension, and prevent the hydrocephalus [8, 9].

Lumbar cistern drainage is a commonly used approach [10-12], which has been widely used in clinics, especially for patients who receive intravascular interventional therapy. However, for patients with high intracranial pressure or intracranial haematoma, there is a risk of induced brain herniation in the lumbar cistern drainage, especially for patients who have completed the craniotomy for aneurysm clipping. The intracranial pressure further increases due to surgical trauma and brain oedema [13-17]. Patients who have undergone the craniotomy, intraoperative placement of carotid cistern drainage tubes and continuous drainage after the operation are performed. This is effective in the removal of the subarachnoid haemorrhagic CSF. Because the carotid cistern drainage has no transfer factors from the supratentorial pressure to the infratentorial pressure, the risk of cerebral herniation induced by lumbar cistern drainage is avoided. The lumbar cistern drainage tube products used in the present study were manufactured by Medtronic. These tubes are fine and soft, with very little or almost no stimulation to the peripheral nerves and vessels when they are placed in the carotid cistern.

In the clinical application in 6 patients, the pressure of the carotid cistern drainage tube was managed within $80-180 \mathrm{~mm} \mathrm{Hg}$. No reactions of oculomotor nerve paralysis, vision loss, heart rate and blood pressure changes were reported. During the carotid cistern drainage, these patients had no significant headache, or their pain scores were within 1-6 points [18]. The postoperative intracranial pressure was usually low on the day of the operation, ranging within $0-10 \mathrm{~mm} \mathrm{Hg}$, or even had a negative value. This may be correlated to the CSF excretion and the presence of intracranial air during the operation, or because the postoperative subcutaneous drainage tube's drainage bag hangs at the bedside, producing a siphon action and resulting in negative pressure drainage. After the removal of the subcutaneous drainage tube, the pressure of the carotid cistern drainage tube is managed within the range $80-180 \mathrm{~mm} \mathrm{Hg}$. The intracranial pressure gradually recovered from the day of the operation and from the presence of the subcutaneous drainage tube. The median intracranial pressure of each patient ranged within 6-16 mm Hg. Compared with the patients without carotid cistern drainage, it shows that carotid cistern drainage has a significant effect in controlling intracranial pressure.

Because the monitoring time of intracranial pressure is basically the same as the drainage time of the carotid cistern in this group of patients, the intracranial pressure monitor was withdrawn, and the drainage tube of the carotid cistern was removed. There are no data showing whether the intracranial pressure gradually increases after the drainage of the carotid cistern is stopped in this group of patients. However, the pain scores of these patients increased after the removal of the drainage tube of the carotid cistern, according to the patients' numeric scores on pain. These patients had low pain scores, within 1-6 points. This suggests that the drainage of the carotid cistern could reduce the stimulation of blood and relieve the headache by reducing intracranial hypertension after removing the haemorrhagic CSF. The level of pain increases after stopping the carotid cistern, but remains within the mild-to-moderate range. This may be associated with timely clearance of subarachnoid haemorrhagic CSF. The asymptomatic cerebral vasospasm and hydrocephalus are seen in this group of patients who are followed up for 3-6 months. The prognosis is good and the GOS scores are in grade 5 .

There are limitations to the present study. Firstly, the present study was a real-world case report study rather than a case-controlled study. Therefore, there is still a certain risk of bias. Secondly, the present study was a single-centre clinical study, and the sample size was small. Thus, there is a need to further increase the sample size and carry out multicentre clinical studies. Thirdly, the present study only introduced the methods for the carotid cistern drainage of haemorrhagic CSF and the objective data of this group of patients. Due to the small sample size, statistical conclusions for the lumbar cistern drainage could not be drawn, or there were no drainage controls. These corresponding conclusions need to be drawn after further accumulation of data. Fourthly, the removal of carotid cistern drainage 24 to $72 \mathrm{~h}$ after surgery is our only method at this stage. The duration of drainage is assessed based on the amount of subarachnoid haemorrhage (CT Fisher's grade), intracranial pressure monitoring junction, and the patient's symptoms. The duration of drainage tube placement needs to be further studied clinically.

In conclusion, the CSF drainage of the carotid cistern applied for patients with aneurysmal subarachnoid haemorrhage during craniotomy for aneurysm clipping can effectively remove the subarachnoid haemorrhage, control the intracranial hypertension, and reduce the incidence of cerebral vasospasm and hydrocephalus. This plays a positive role in preventing CSF leakage and improving the prognosis.

\section{Conflict of interest}

The authors declare no conflict of interest. 


\section{References}

1. Petridis AK, Kamp MA, Cornelius JF, et al. Aneurysmal subarachnoid hemorrhage. Dtsch Arztebl Int 2017; 114: 226-36.

2. van Donkelaar CE, Bakker NA, Veeger NJ, et al. Predictive factors for rebleeding after aneurysmal subarachnoid hemorrhage: rebleeding aneurysmal subarachnoid hemorrhage study. Stroke 2015; 46: 2100-6.

3. Molyneux AJ, Birks J, Clarke A, Sneade M, Kerr RS. The durability of endovascular coiling versus neurosurgical clipping of ruptured cerebral aneurysms: 18 year follow-up of the UK cohort of the International Subarachnoid Aneurysm Trial (ISAT). Lancet 2015; 385: 691-7.

4. Lindgren A, Vergouwen MD, van der Schaaf I, et al. Endovascular coiling versus neurosurgical clipping for people with aneurysmal subarachnoid haemorrhage. Cochrane Database Syst Rev 2018; 8: CD003085.

5. Wolf S. Rationale for lumbar drains in aneurysmal subarachnoid hemorrhage. Curr Opin Crit Care 2015; 21: 120-6.

6. Nath S, Koziarz A, Badhiwala JH, et al. Predicting outcomes in aneurysmal subarachnoid haemorrhage. BMJ 2018; 360: k102.

7. Rabinstein AA, Lanzino G. Aneurysmal subarachnoid hemorrhage: unanswered questions. Neurosurg Clin N Am 2018; 29: 255-62.

8. Kumar G, Shahripour RB, Harrigan MR. Vasospasm on transcranial Doppler is predictive of delayed cerebral ischemia in aneurysmal subarachnoid hemorrhage: a systematic review and meta-analysis. J Neurosurg 2016; 124: 1257-64.

9. Burns SK, Brewer KJ, Jenkins C, Miller S. Aneurysmal subarachnoid hemorrhage and vasospasm. AACN Adv Crit Care 2018; 29: 163-74.

10. Xu N, Meng H, Liu T, Feng Y, Qi Y, Wang H. Continuous lumbar cistern drainage before surgical clipping for aneurysmal subarachnoid hemorrhage. World Neurosurg 2018; 26. pii: S1878-8750(18) 30107-4.

11. Chen QH, Lin D, Yu QG, Zhou J. Efficacy of lumbar cistern drainage combined with intrathecal antibiotherapy for the treatment of ventriculo-subarachnoid infections following surgery for hypertensive intracerebral hemorrhage. Neurochirurgie 2016; 63: 13-6.

12. Diringer MN, Zazulia AR. Aneurysmal subarachnoid hemorrhage: strategies for preventing vasospasm in the intensive care unit. Semin Respir Crit Care Med 2017; 38: 760-7.

13. Bardutzky J, Witsch J, Juttler E, Schwab S, Vajkoczy P Wolf S. EARLYDRAIN - outcome after early lumbar CSF-drainage in aneurysmal subarachnoid hemorrhage: study protocol for a randomized controlled trial. Trials 2011; 12: 203

14. Kim YS, Kim SH, Jung SH, Kim TS, Joo SP. Brain stem herniation secondary to cerebrospinal fluid drainage in ruptured aneurysm surgery: a case report. Springerplus 2016; 5: 247.

15. Tangen K, Narasimhan NS, Sierzega K, Preden T, Alaraj $A$, Linninger $A A$. Clearance of subarachnoid hemorrhage from the cerebrospinal fluid in computational and in vitro models. Ann Biomed Eng 2016; 44: 3478-94.

16. Cossu G, Messerer M, Stocchetti N, Levivier M, Daniel RT, Oddo $M$. Intracranial pressure and outcome in critically ill patients with aneurysmal subarachnoid hemorrhage: a systematic review. Minerva Anestesiol 2016; 82: 684-96.
17. Magni F, Pozzi M, Rota M, Vargiolu A, Citerio G. High-resolution intracranial pressure burden and outcome in subarachnoid hemorrhage. Stroke 2015; 46: 2464-9.

18. Čomić H, Rinkel GJE, Vergouwen MDI. The initial timecourse of headache in patients with spontaneous subarachnoid hemorrhage. J Neurol Sci 2017; 379: 55-7. 